\title{
STATE EMPLOYMENT POLICY IN THE CONDITIONS OF THE COVID-19 PANDEMIC CRISIS IN GEORGIA
}

\author{
IRMA TKEMALADZE \\ PhD Student \\ Ivane Javakhishvili Tbilisi State University, Georgia \\ irma.tkemaladze@tsu.ge
}

\begin{abstract}
The crisis caused by the Covid-19 pandemic has not only economic character, it is multifaceted, so ways to tackle the problem are more complex and ambiguous. Measures to protect the health of the population often comes into conflict with economic recovery measures, which raises the issue of their proper balancing. At the same time, financial, moral and material support should be provided to the vulnerable sections of the society, at the same time, the party creating the demand for labor should be encouraged to maintain jobs. Recovering the economy requires a comprehensive and consistent strategy based on the interests of each citizen. It is important to have an active social dialogue between employers 'and entrepreneurs' organizations and employees. These measures are relatively easy to implement for countries with high incomes and developed economies, while for low-income countries, the use of fiscal and monetary policy instruments is limited and therefore becomes a more difficult process. In such a case, it is recommended to differentiate the directions of the state employment policy according to the priority. The first line of action in the conditions of the covid-19 pandemic should be to stop the growth of poverty caused by declining employment income from reduced employment. As mentioned above, the government's anti-crisis plan even provides for passive policy measures and appropriate funding. However, it should be noted that the simplification of bureaucratic procedures related to the issuance and administration of targeted benefits would also be desirable. The activation of passive policy has accelerated the "formalization" of informal employees, it is recommended to continue working in this direction through a different prism to prevent the growth of precariate in the labor market. It is advisable to increase the number of jobs in the formal sector, to adequately remunerate the qualifications and energy expended, to create normal working conditions and to provide real protection of the labor rights of the employees. It is recommended to finance various projects and startups to promote self-employment and motivate the unemployed people. An important measure is the systematic research of labor market needs, which creates a clear picture of the requirements in the field of employment in the policy-making process and is a vector in the planning of training relevant qualified personnel. The latter will help to eliminate the most important problems of the Georgian labor market - the imbalance between labor demand and supply.
\end{abstract}

KEYWORDS: LABOR MARKET, EMPLOYMENT, REMOTE EMPLOYMENT, UNEMPLOYMENT, PANDEMIC CRISIS.

For citation: Tkemaladze, I. (2021). State Employment Policy in The Conditions of The Covid-19 Pandemic Crisis in Georgia. Globalization and Business. 12, 155-161. (In Georgian). https://doi.org/10.35945/gb.2021.12.021 


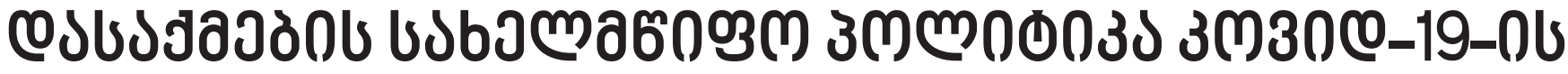

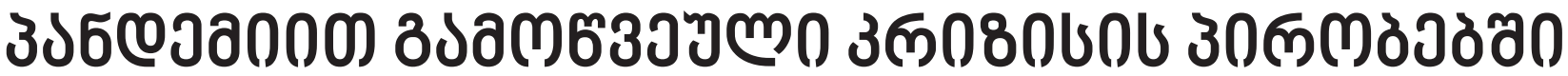

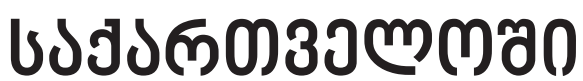

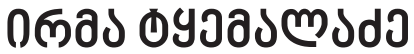

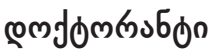

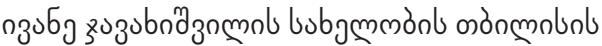

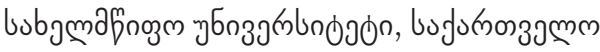 \\ irma.tkemaladze@tsu.ge
}

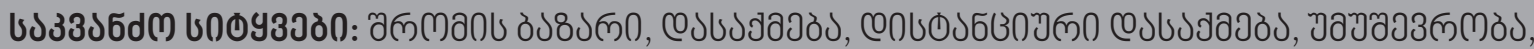

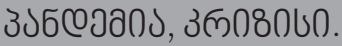

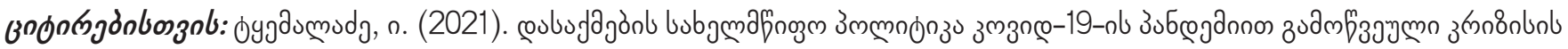

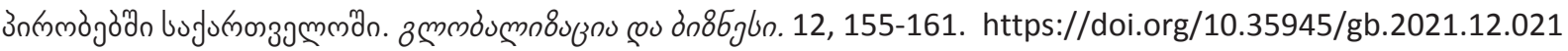

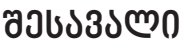

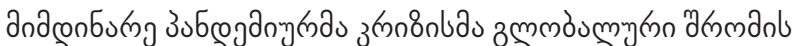
зu8s

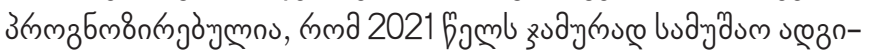

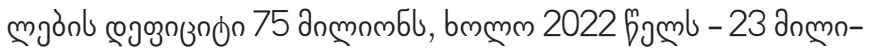
mbl 8১yommegojos (World Employment and Social Outlook:

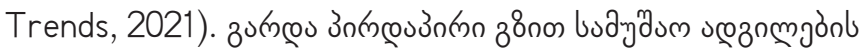

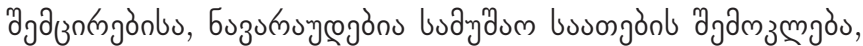

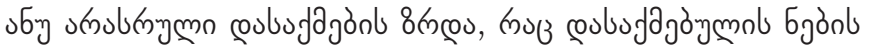

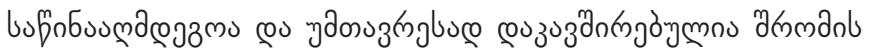

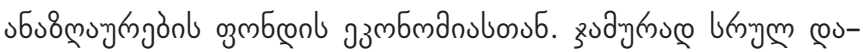

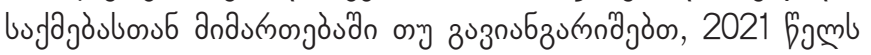

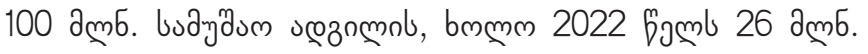

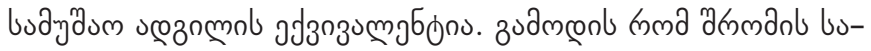

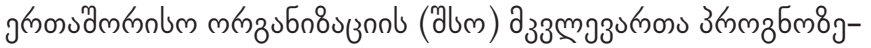

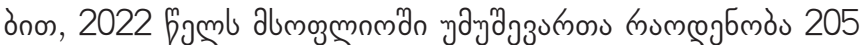

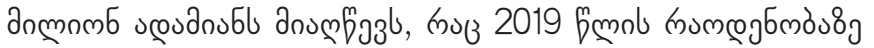

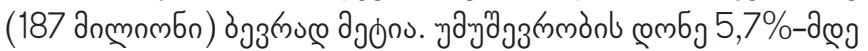

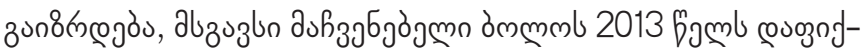

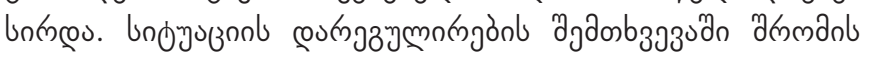

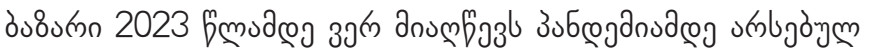

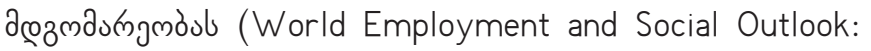
Trends, 2021).

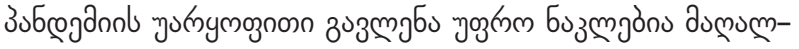

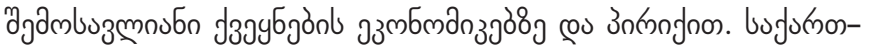

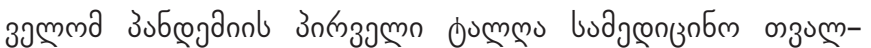

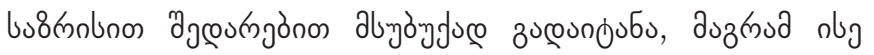

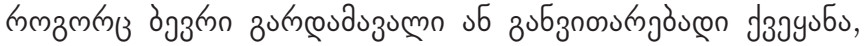

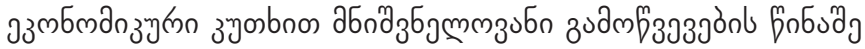

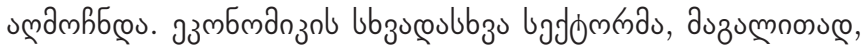

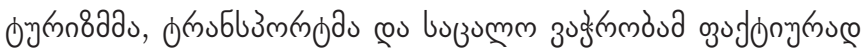

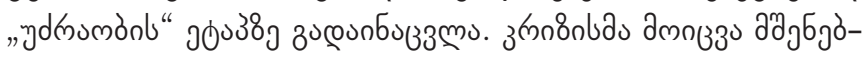

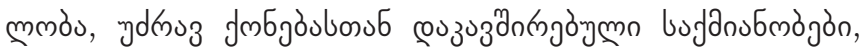
bəмmलзб

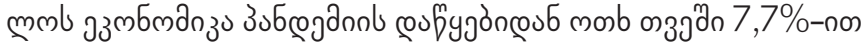

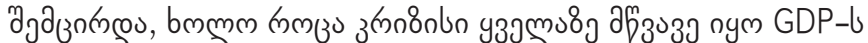

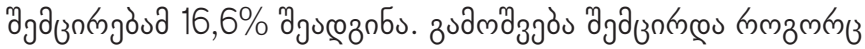

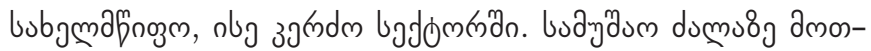

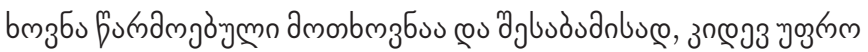

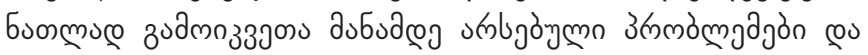
उuhb ang r zudu

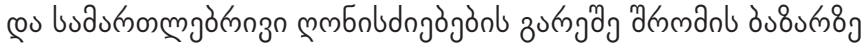

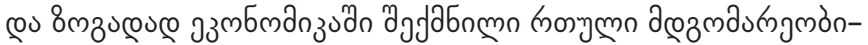

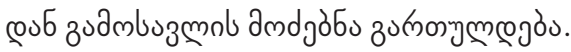

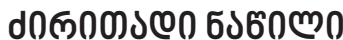

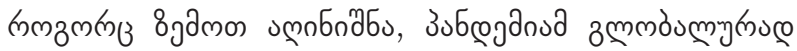

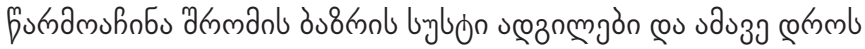

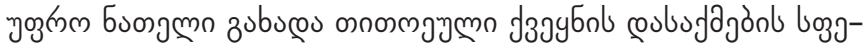

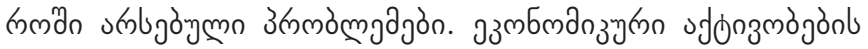

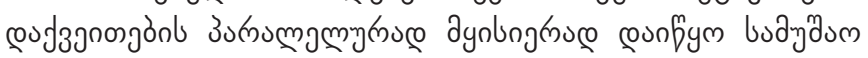

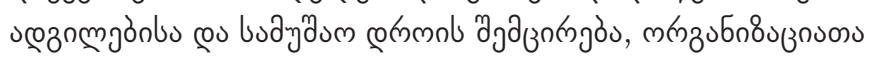




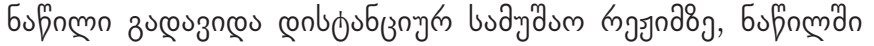

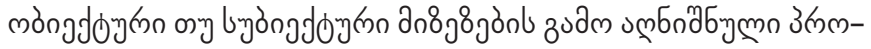

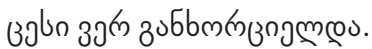

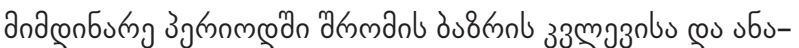

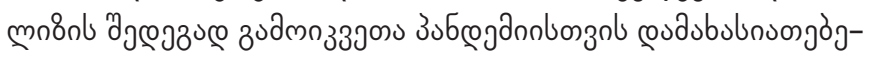

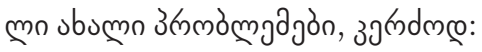

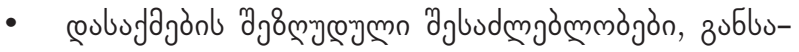

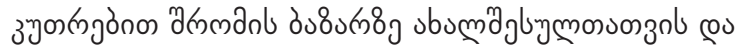
jumgònbsonzol;

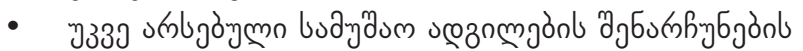
augumo molizo;

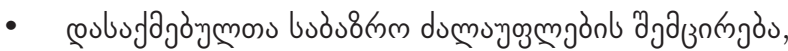

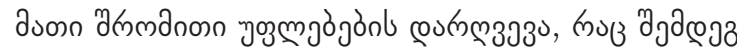
дmз зलлб

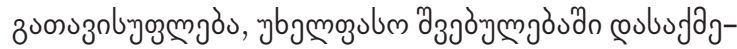

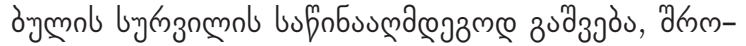

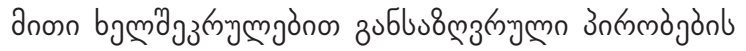

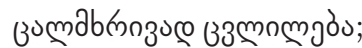

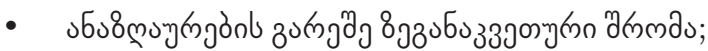

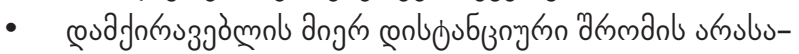

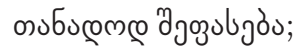

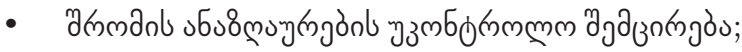

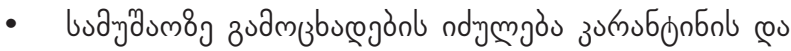

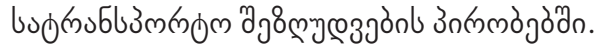

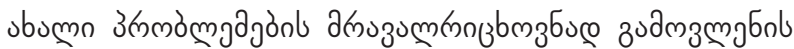

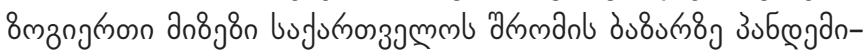

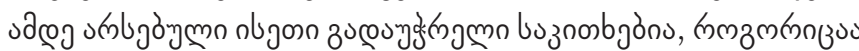

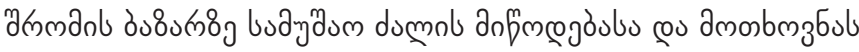

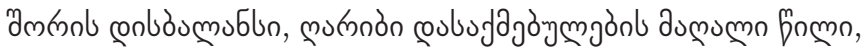

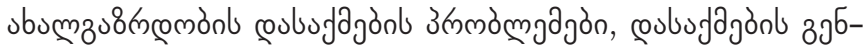

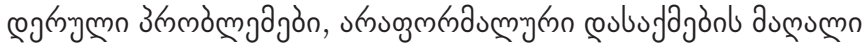

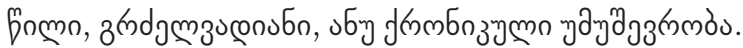

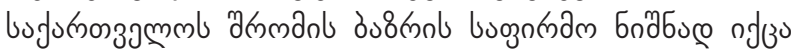

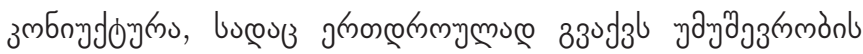

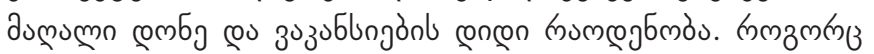

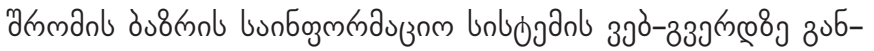

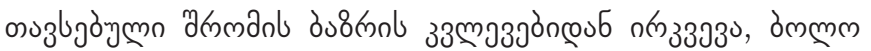

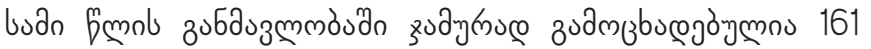

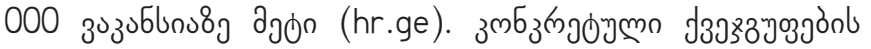

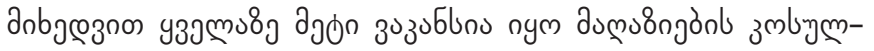

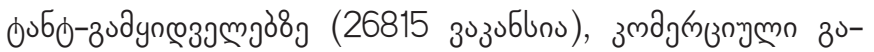

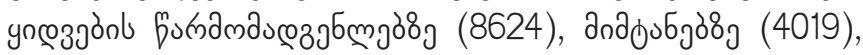

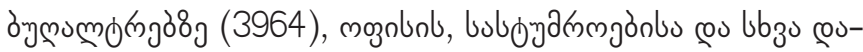
f fl

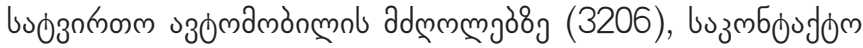

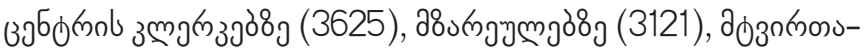

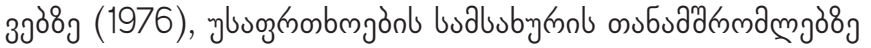

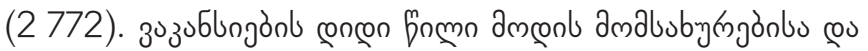

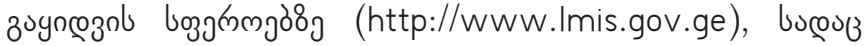

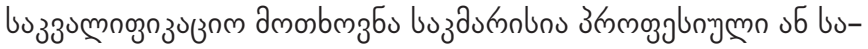

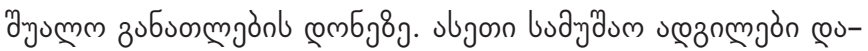

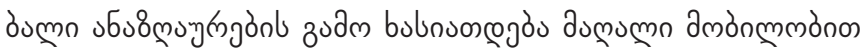

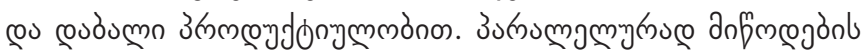

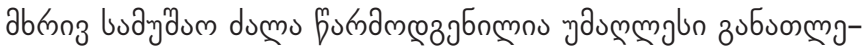

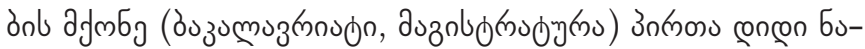

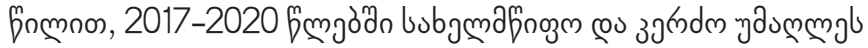

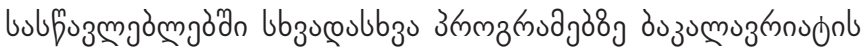

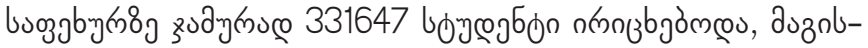

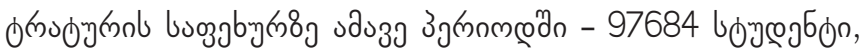

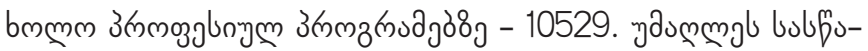

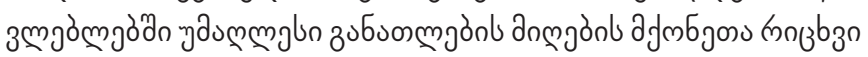

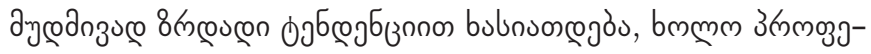

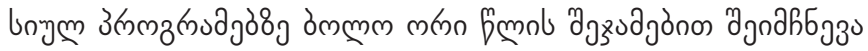

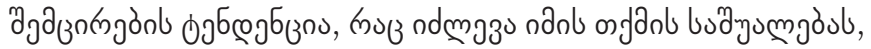

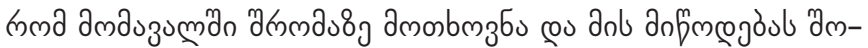

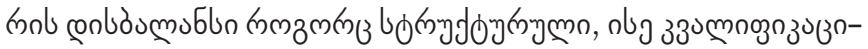

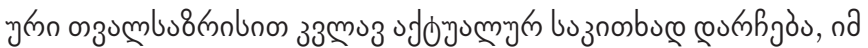

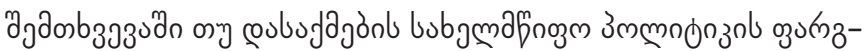

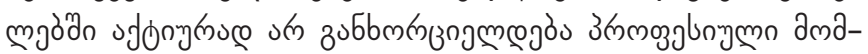

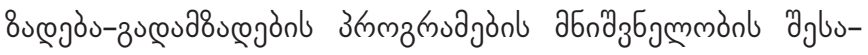

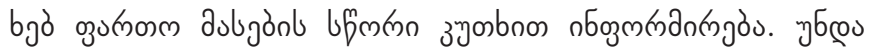

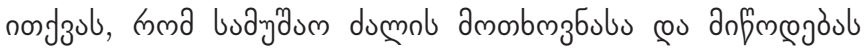

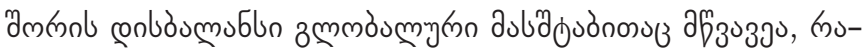

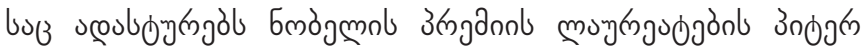

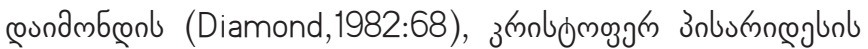
(o) egnm dmrnogblgbols (Mortensen \& Pissarides, 1994) пбол

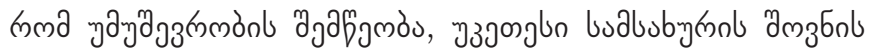

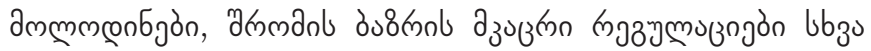

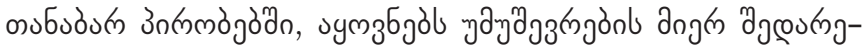

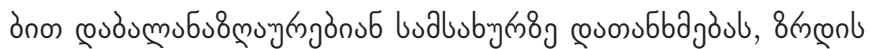

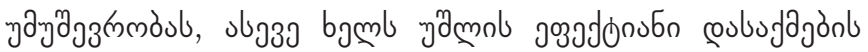
unigòmóul.

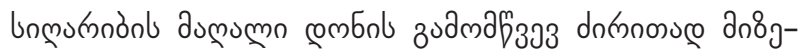

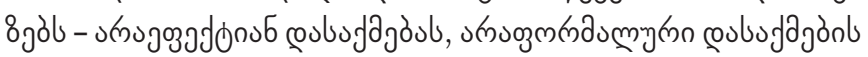

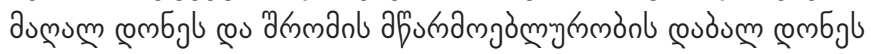

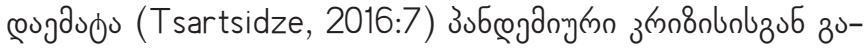

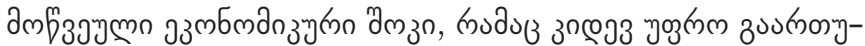

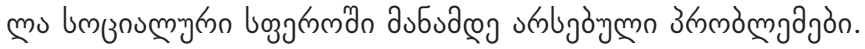

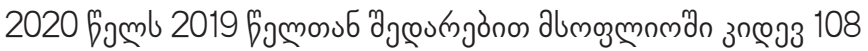

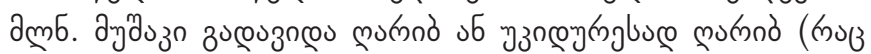

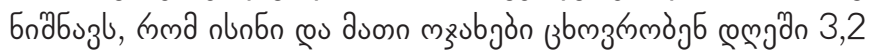

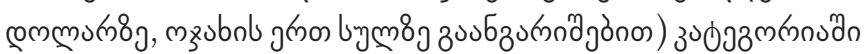
(World Employment \& Social Outlook: Trends, 2021). noof

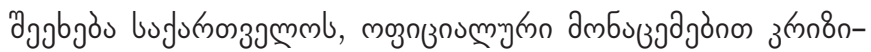

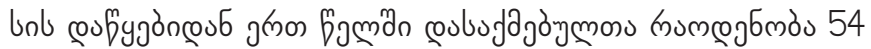

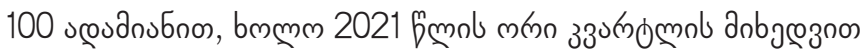

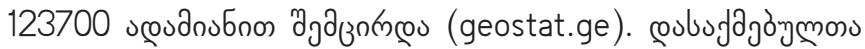




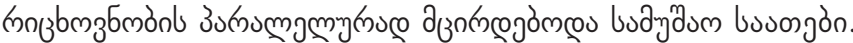

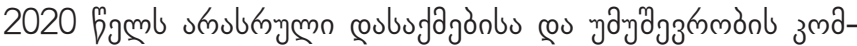

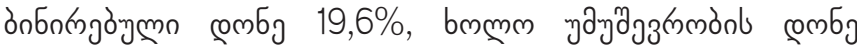

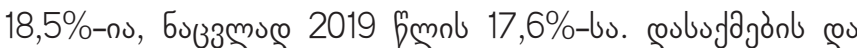

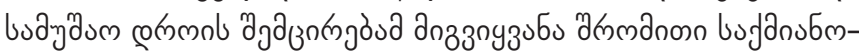

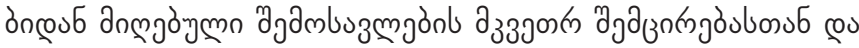

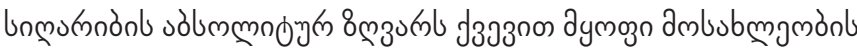
fromoll 8 mpoulonst.

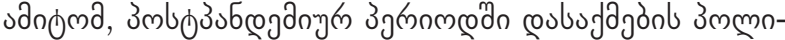

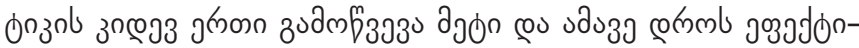

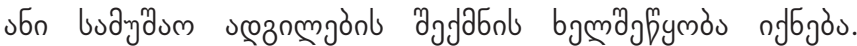

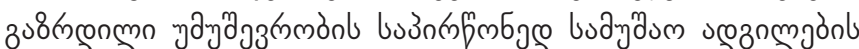

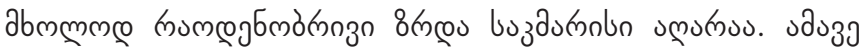

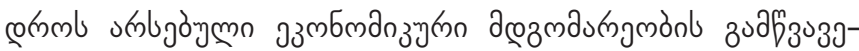
on muznç

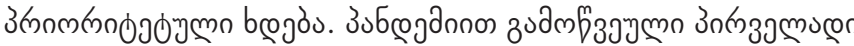

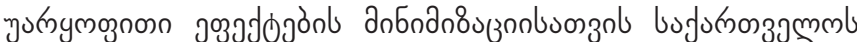

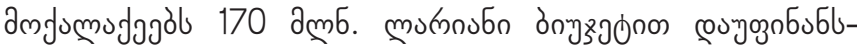

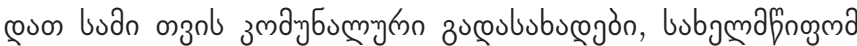

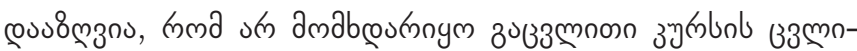

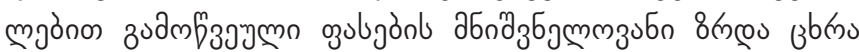

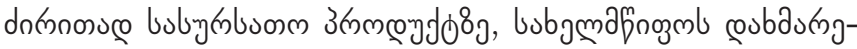

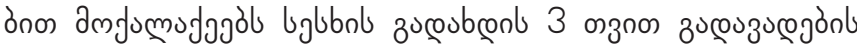

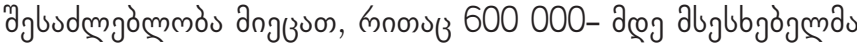

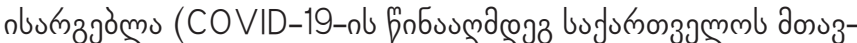

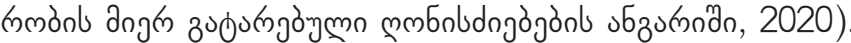

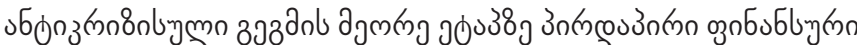

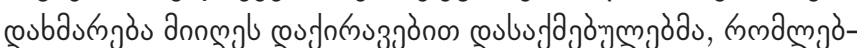

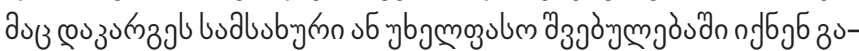

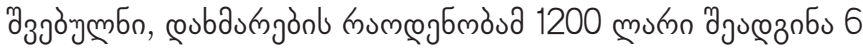

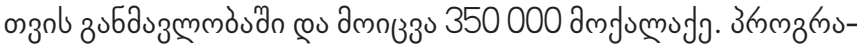

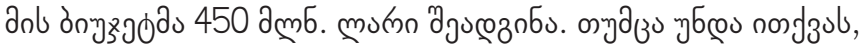

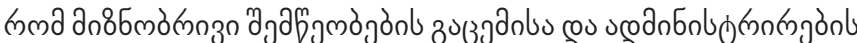

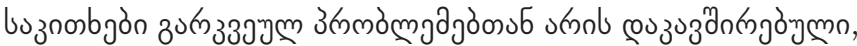

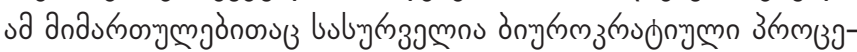

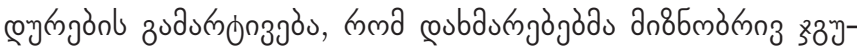

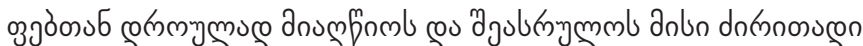

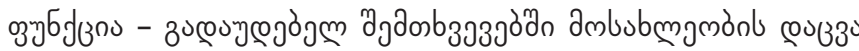

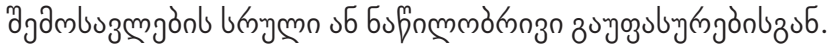

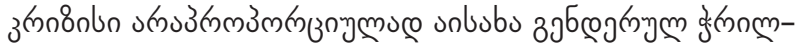

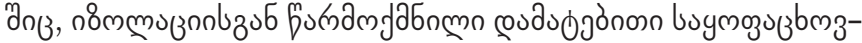

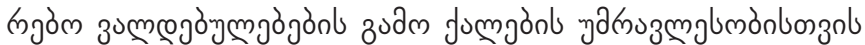

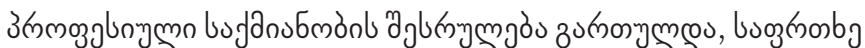
गjgdabo e

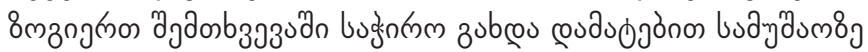

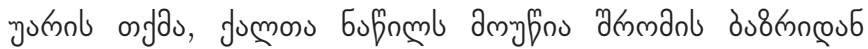

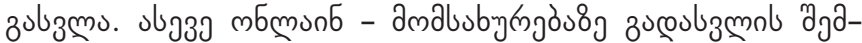

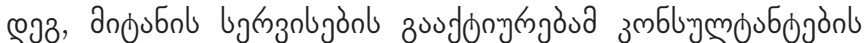

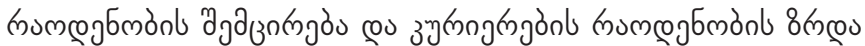

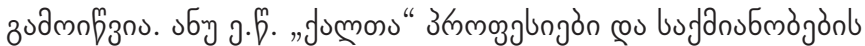
פa

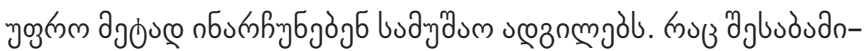

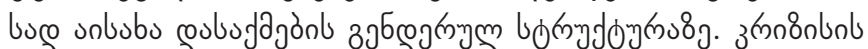

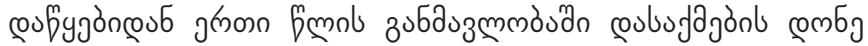

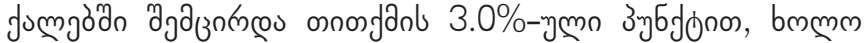

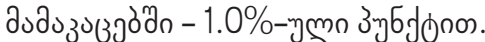

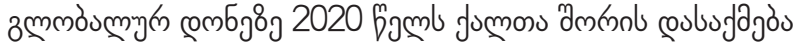

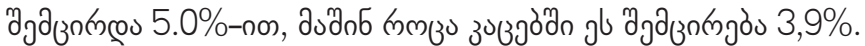

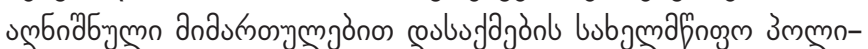
jnzol zмб з з

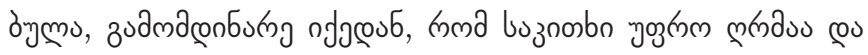

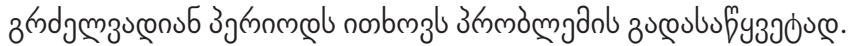

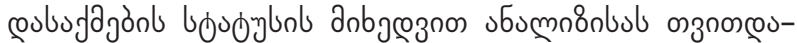

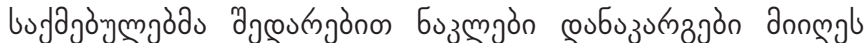

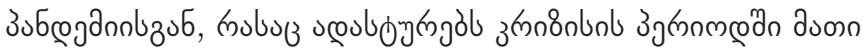

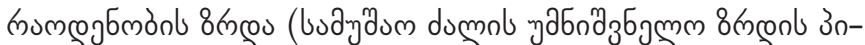

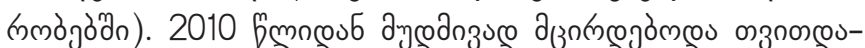

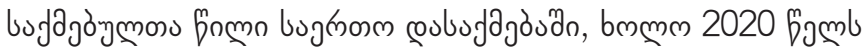

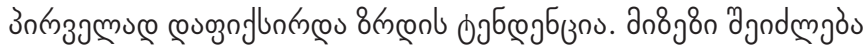

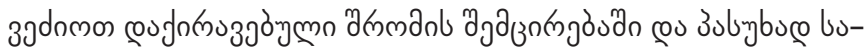

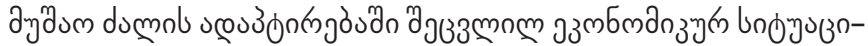

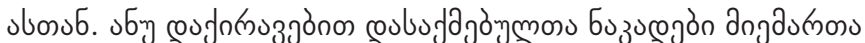

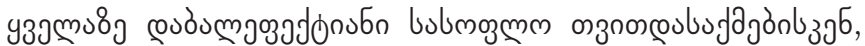

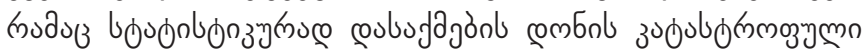

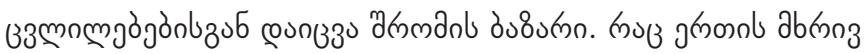

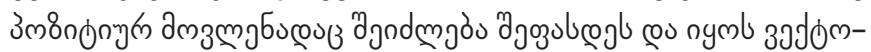

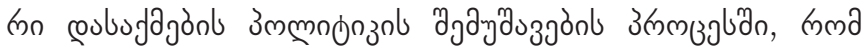

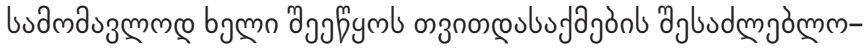

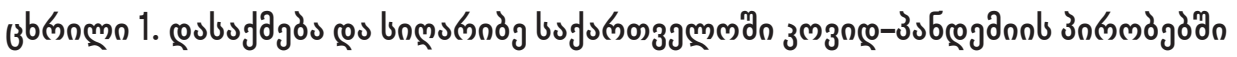

\begin{tabular}{|c|c|c|}
\hline as & 2019 & 2020 \\
\hline 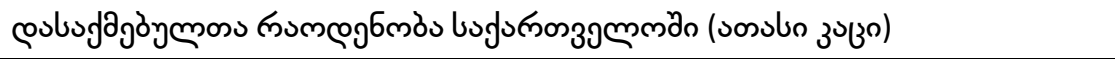 & 1295.9 & 1241.8 \\
\hline 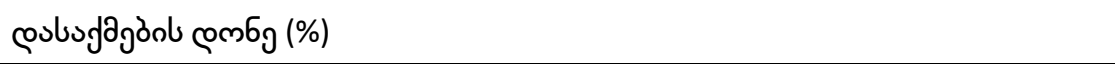 & 42,67 & 41,14 \\
\hline 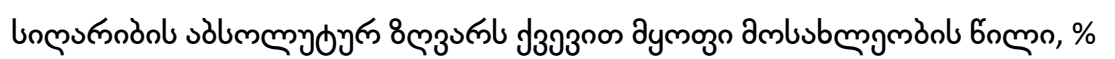 & 19,5 & 21,3 \\
\hline
\end{tabular}

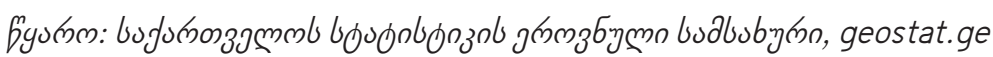




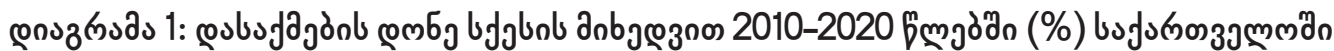

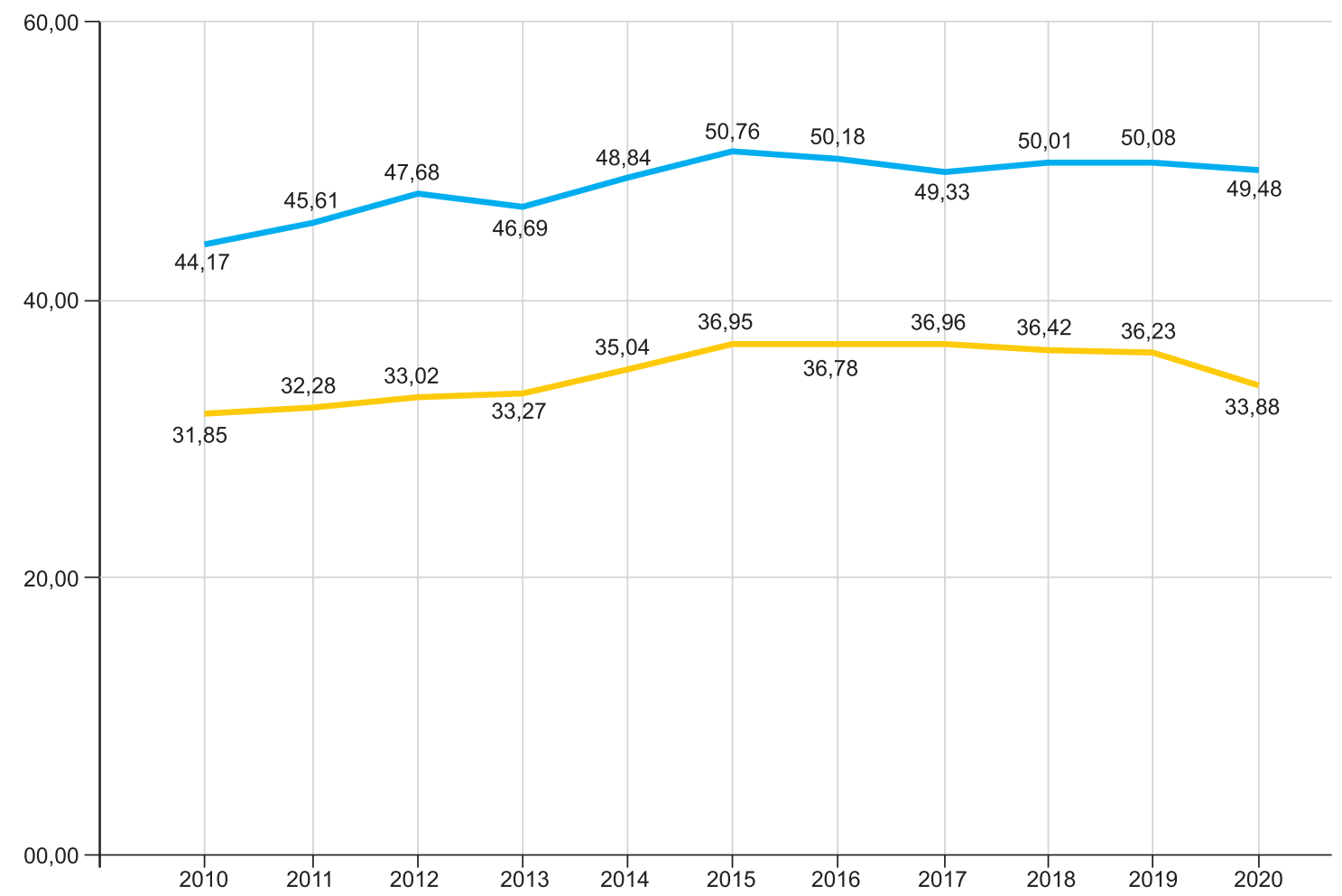

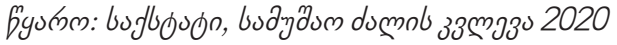

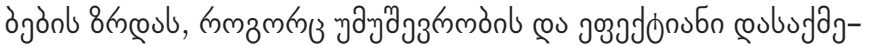

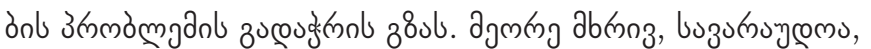

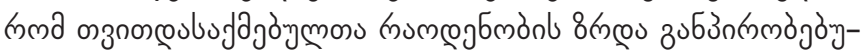

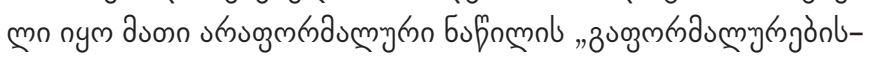

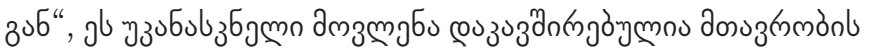

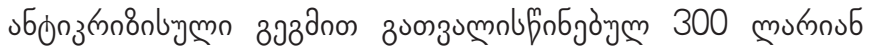

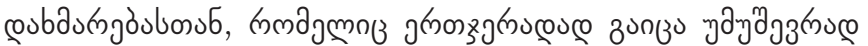

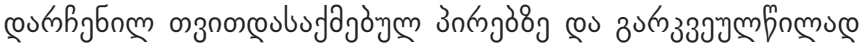

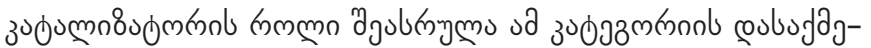

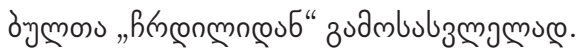

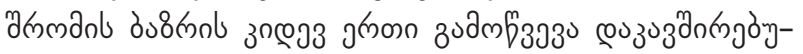

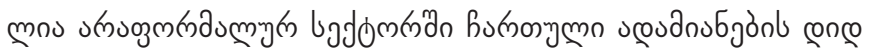

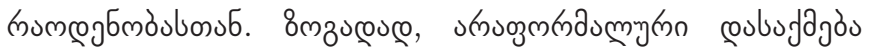

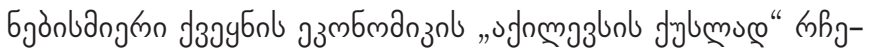

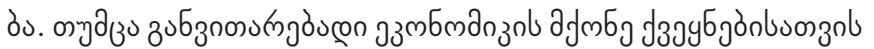

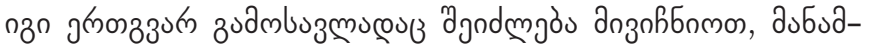

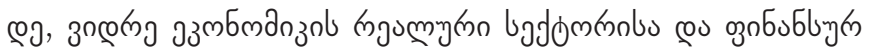

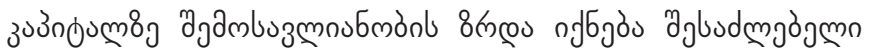
(Kakulia \& Chikobava, 2016;123).

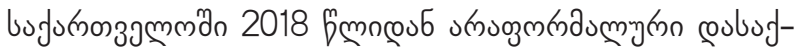

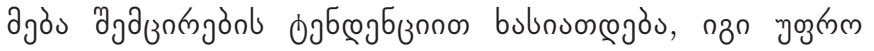

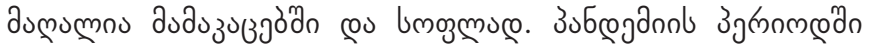

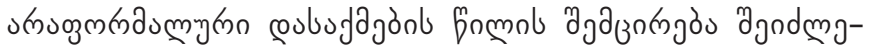

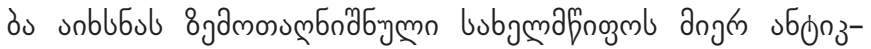

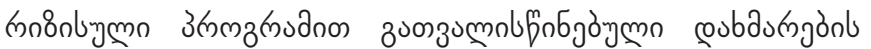

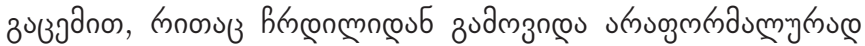

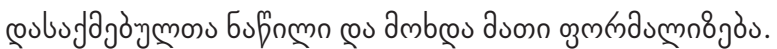

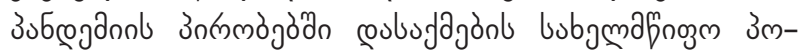

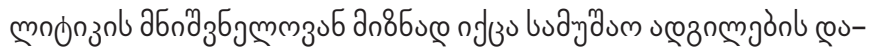

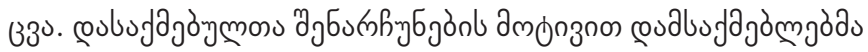

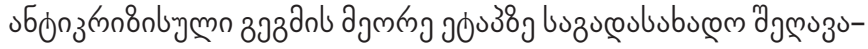

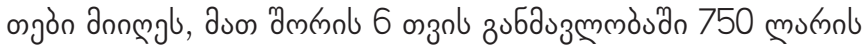

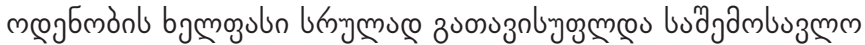

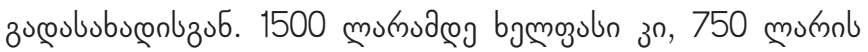

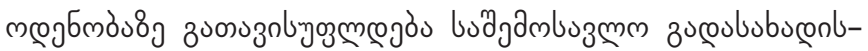

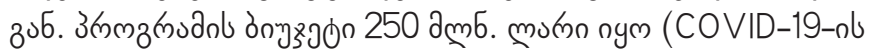

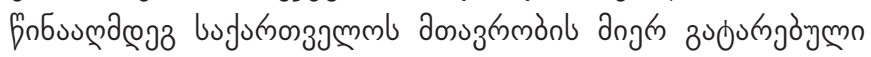

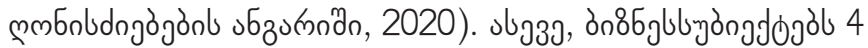

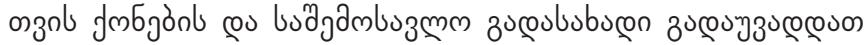

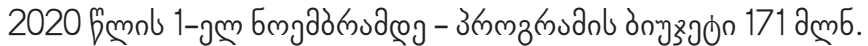

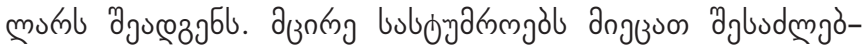

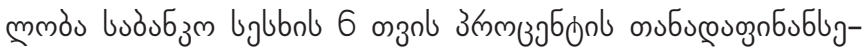

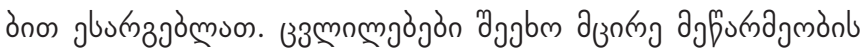

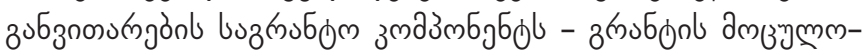

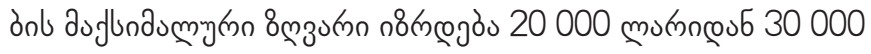

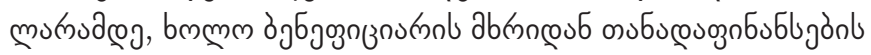

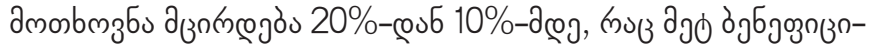
u们

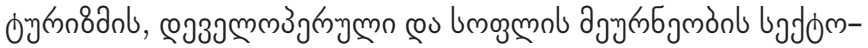

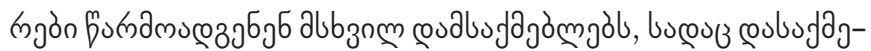




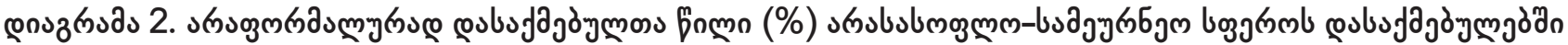

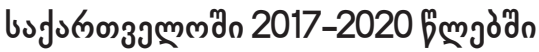

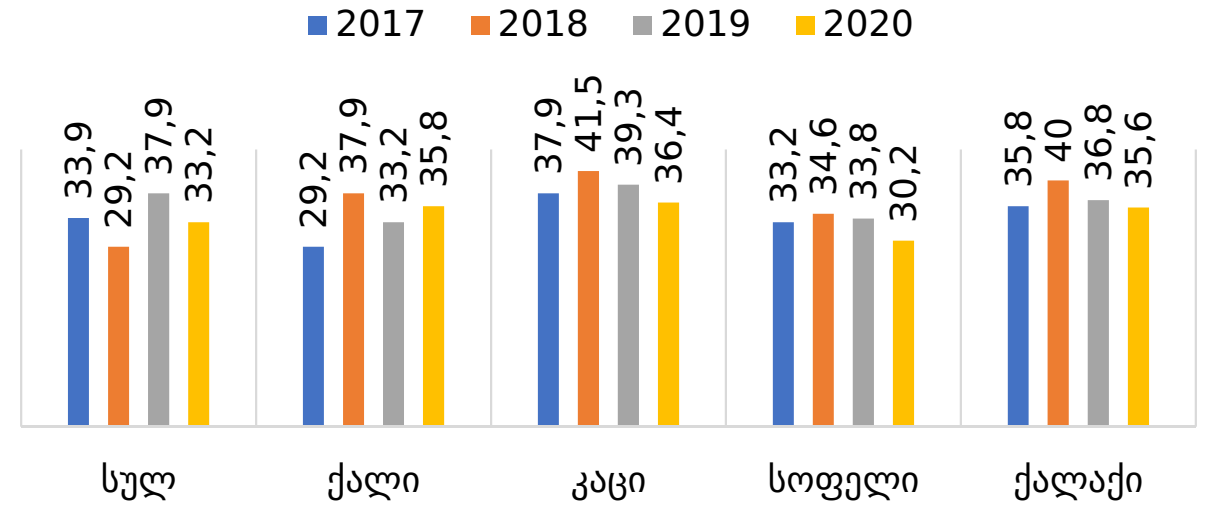

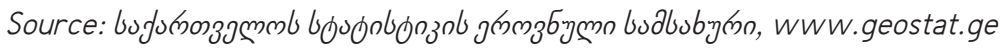

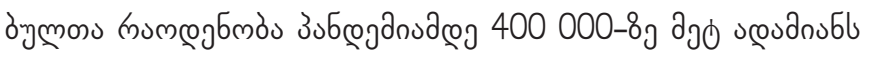

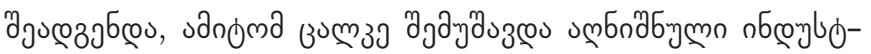

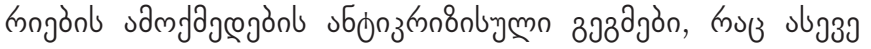

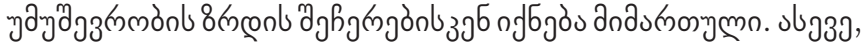

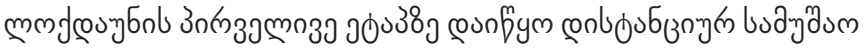

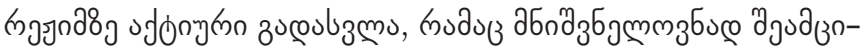

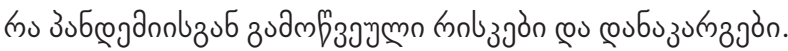

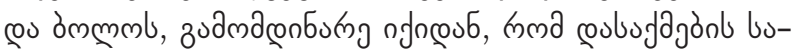

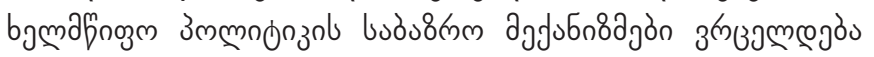

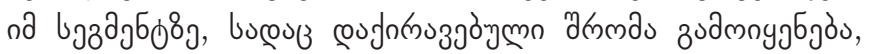

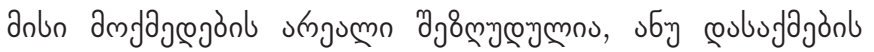

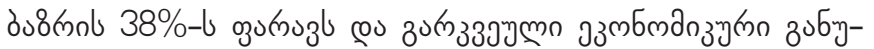
bu8şз चुaznn

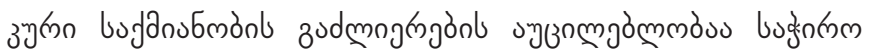

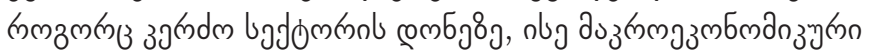

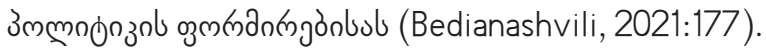

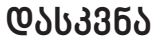

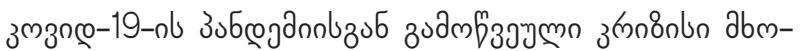

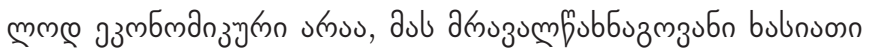

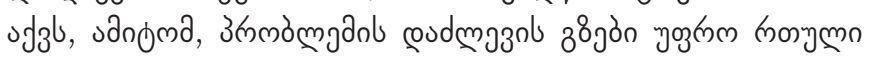

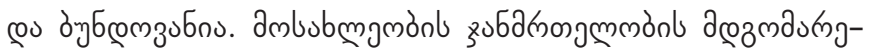

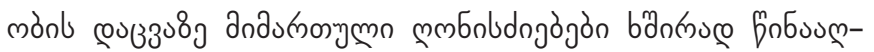

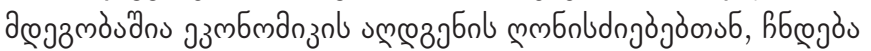

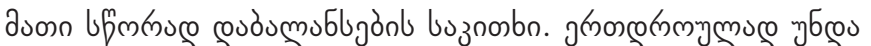

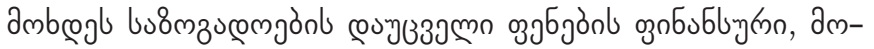

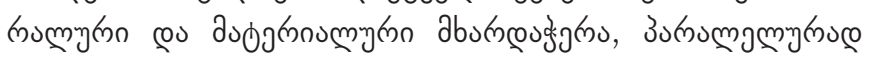

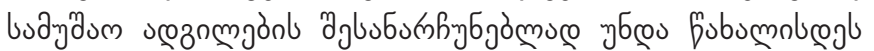

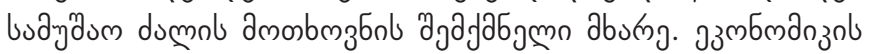

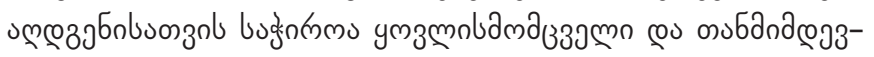

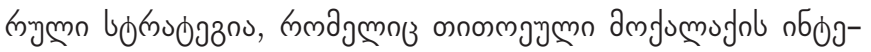

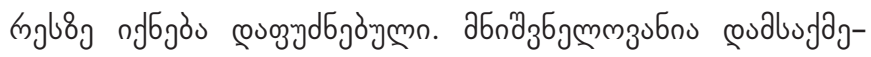

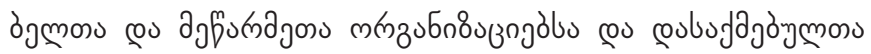

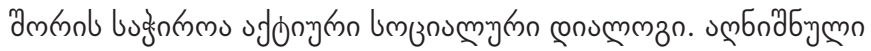

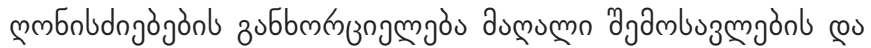

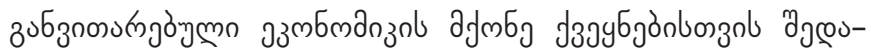

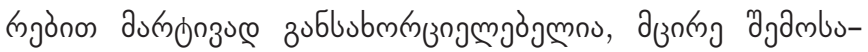
з

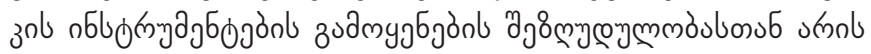

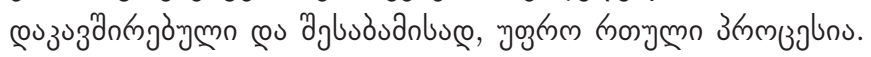

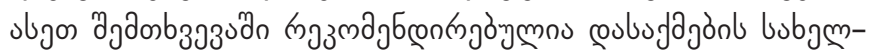

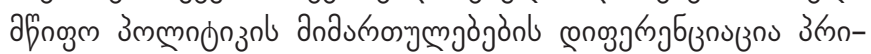

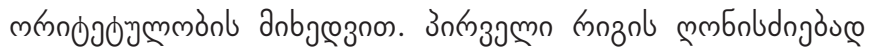

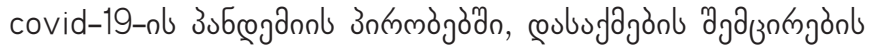

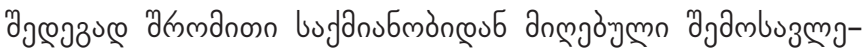

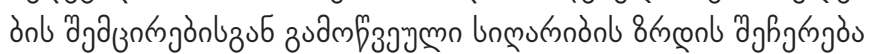

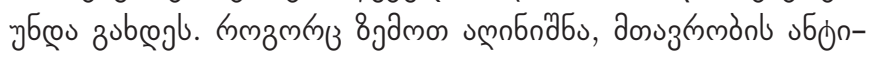

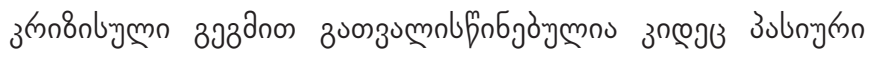

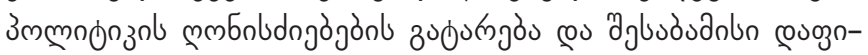

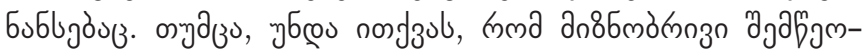

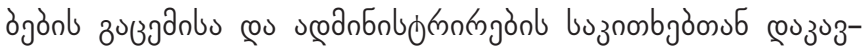

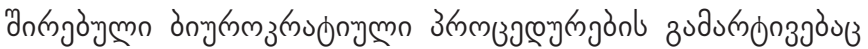

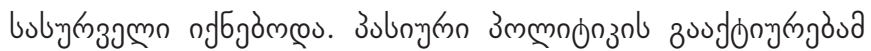

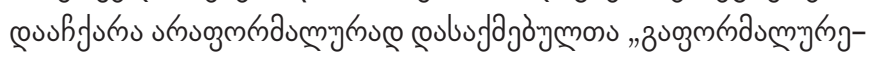

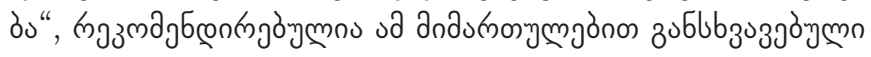

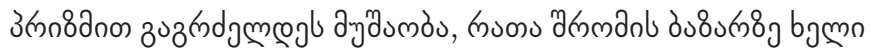
ajgàmel 3

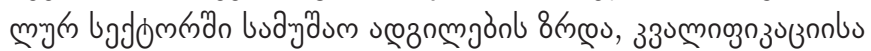

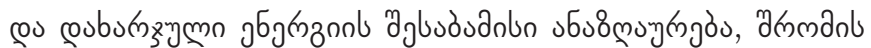

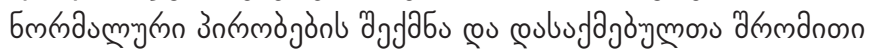

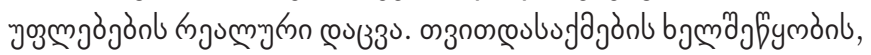

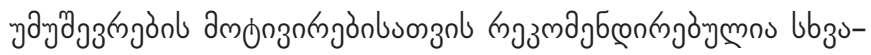

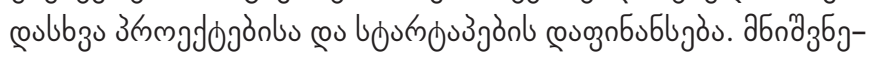

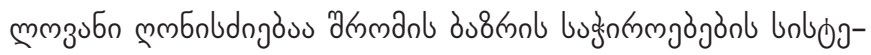

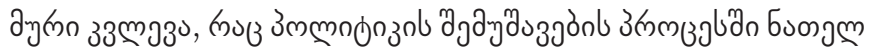




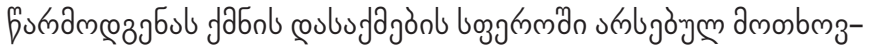

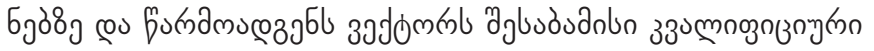

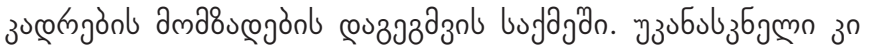

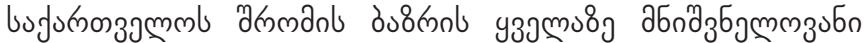

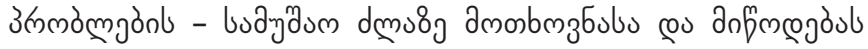

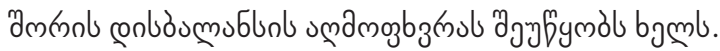

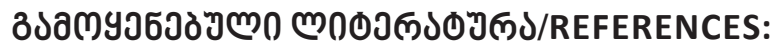

Bedianashvili, G. (2018). Culture as a factor of knowledge economics with paradigmatic changes in systemic institutional context. Globalization and Business, 6, 59-62 (In Georgian).

Bedianashvili, G. (2021). Macroeconomic and Cultural Determinants of the COVID-19 Pandemic Crisis. Bulletin of the Georgian National Academy of Sciences, 15(2), 191-197.

Data of the National Statistics Office of Georgia - https://www.geostat.ge/ka (In Georgian).

Diakonidze, A., \& Natsvlishvili, V. (2020). Labor and Social Security during the New Coronavirus Pandemic. Open Society Fund (In Georgian).

Diamond, P. (1982). Aggregate demand management in search equilibrium. Journal of Political Economy.

Enterprise Demand Survey on Skills. (2019). Tbilisi: Ministry of Economy and Sustainable Development of Georgia (In Georgian). Georgian Labor Market Survey. (2020). Tbilisi: Ministry of Economy and Sustainable Development of Georgia (In Georgian).

http://mes.gov.ge/content.php?lang=geo\&id=1028

https://ec.europa.eu/eurostat

Kakulia, N., \& Chikobava, M. (2016). The possibility of Overcoming the Current Economic Crisis through the Use of Negative Interest Rates, Ivane Javakhishvili Tbilisi State University, The collection of The International Scientific-Practical Conference Materials, 125-132 (In Georgian).

Kakulia, N., \& Lazarashvili, T. (2019). Institutional transformation and its challenges in Georgia. Ivane Javakhishvili Tbilisi State University, The collection of The 4th International Scientific-Practical Conference Materials, 173-178 (In Georgian).

Law of Georgia N6819-SR On Employment Promotion, 2020 years, 14 Juile (In Georgian).

Papava, V. (2020). On the COVID-19 Pandemic Economic Crisis and the Reorientation of the Georgian Economy, Reflecting on a Changed World, 215-221.

Report on the measures taken by the Government of Georgia against COVID-19. (2020). (In Georgian).

Resolution of the Government of Georgia N662 On Approval of the National Strategy of Labor and Employment Policy of Georgia for 2019-2023. (2019). (In Georgian).

Saltiel, F. (2020). Who Can Work From Home in Developing Countries? working paper, Duke University, Durham, North Carolina.134-168

Silagadze, A., Atanelishvili, \& T., Silagadze, N. (2020). Some Tendencies of Post-Soviet Migration and Unemployment. Bulletin of the Georgian National Academy of Sciences, 14 (1), 175-176. (In Georgian).

Tsartsidze, M. (2019). Active labor market policy and peculiarities of youth employment in Georgia. Ekonomics and Business, XI (2), 16-37. (In Georgian).

Tsartsidze, M. (2019). Efective Employment - An important factor in human well-being and economic growth in Georgia. Economisti, 2, 79-82. (In Georgian).

Tukhashvili, M., \& Shelia, M. (2012). The impact of labor emigration on the demographic and economic development of Georgia in the post-Soviet period. http://www.carim-east.eu/media/CARIM-East-2012-RR-29.pdf.

World Economic Outlook, (2020). IMF. https://www.imf.org/en/Publications/WEO-/Issues/2020/04/14/weo-april-2020

World Employment and Social Outlook: Trends (2021), ILO. International Labour Office - Geneva. https://www.ilo.org/global/ research/global-reports/weso/trends2021/WCMS_795453/-lang--en/index.htm 